\title{
CLINICAL EXPERIENCE WITH ONE HUNDRED CONSECUTIVE PATIENTS UNDERGOING ORTHOTOPIC HEART TRANSPLANTATION WITH BICAVAL AND PULMONARY VENOUS ANASTOMOSES
}

Alfredo Trento, $\mathrm{MD}^{\mathrm{a}}$

Johanna M. Takkenberg, MD

Lawrence S. C. Czer, MD

Carlos Blanche, $\mathrm{MD}^{\mathrm{a}}$

Sharon Nessim, DrPH ${ }^{c}$

Mabelle H. Cohen, BA ${ }^{b}$

Robert Kass, MD

Sharo Raissi, $\mathrm{MD}^{\mathrm{a}}$

Jack M. Matloff, MD
Objective: Our objective was to assess survival, need for pacemaker insertion, and rejection frequency with a new surgical technique of orthotopic heart transplantation using bicaval and pulmonary venous anastomoses. Methods: We retrospectively reviewed 100 consecutive patients who had orthotopic heart transplantation with this technique between July 1991 and September 1995. Results: The mean age was $57.0 \pm 11.1$ years, with 51 patients being 60 years or older. The mean donor/recipient weight ratio was 0.92 , and in 28 patients the ratio was less than 0.8 . The early (30-day) survival was $100 \%$ and the 1 - and 2-year survivals were $98 \% \pm 2 \%$ and $96 \%$ $\pm 2 \%$, respectively. Survival was not affected by age or by the duration of the OKT3 therapy $(p>0.2$ for each of these parameters). The seven late deaths were due to infection $(n=2)$, graft atherosclerosis $(n=3)$, acute rejection $(n=1)$, and nonspecific graft failure $(n=1)$. No permanent pacemaker was required in the first 6 months after the operation, and all the patients were discharged in normal sinus rhythm. Freedom from treated rejection was significantly greater in patients with 7 days of OKT3 therapy than in patients with 14 days of therapy $(p<\mathbf{0 . 0 0 0 1 )}$. Conclusions: Orthotopic heart transplantation with bicaval and pulmonary venous anastomoses offers an improved alternative to the standard biatrial technique, with a 30-day mortality of $0 \%$ in 100 consecutive patients, excellent intermediate-term survival, and elimination of the need for pacemaker insertion. More normal anatomic configuration and synchronous function of the atria may have contributed to these results. (J Thorac Cardiovasc Surg 1996;112:1496-503)
$\mathrm{T}$ hirty years ago, Shumway, Lower, and Stofer ${ }^{1}$ originally described what has become the standard technique for orthotopic heart transplantation. An observation was subsequently made that the anastomosis of donor and recipient atria creates enlarged atria with an abnormal geometric configuration and associated mitral and tricuspid regurgitation. ${ }^{2,3}$ Recently, an alternative technique of "to-

From the Divisions of Cardiothoracic Surgery, ${ }^{\mathrm{a}}$ Cardiology, ${ }^{\mathrm{b}}$ and Biostatistics, ${ }^{c}$ Cedars-Sinai Medical Center, Los Angeles, Calif.

Read at the Seventy-sixth Annual Meeting of The American Association for Thoracic Surgery, San Diego, Calif., April 28-May 1, 1996.

Received for publication May 6, 1996; revisions requested June 17, 1996; revisions received July 22, 1996; accepted for publication August 30, 1996.

Address for reprints: Alfredo Trento, MD, Cedars-Sinai Medical Center, No. 6215, 8700 Beverly Blvd., Los Angeles, CA 90048.

Copyright (c) 1996 by Mosby-Year Book, Inc.

$0022-5223 / 96 \$ 5.00+0 \quad 12 / 6 / 77671$ tal" orthotopic heart transplantation was described, the essential features of which are complete excision of the recipient atria and donor heart implantation with bicaval end-to-end anastomoses and pulmonary venous anastomoses. ${ }^{4-6}$ This latter technique produces a nearly normal anatomic configuration of the atria and as a consequence offers the potential for more synchronous atrial contraction, elimination of the need for pacemaker implantation, and reduced atrioventricular valve regurgitation.

We report herein our clinical experience with 100 consecutive patients who underwent heart transplantation for which bicaval and pulmonary venous anastomoses were used.

\section{Patients and methods}

Patients. From December 1988 to October 1995, 171 patients underwent orthotopic heart transplantation at our institution. We retrospectively evaluated the 100 consecutive patients in whom the bicaval and pulmonary venous anastomotic technique was used between July 1991 and October 1995. We excluded seven patients in 
whom a modified technique, consisting of bicaval anastomoses for the right atrium and standard atrial anastomosis for the left atrium, ${ }^{7}$ was used.

Surgical technique for donor heart harvesting. The donor's heart is harvested in the usual fashion with some technical modifications. The superior vena cava (SVC) is transected at or above the azygos vein. The inferior vena cava (IVC) is divided at the diaphragmatic reflection. The pulmonary veins are divided at their entrance into the pericardium, and then the cuffs of the superior and inferior pulmonary veins are joined by an incision to create a single left and single right orifice, leaving a bridge of intact posterior left atrial wall between them.

Surgical technique for recipient. Arterial cannulation is done in the standard fashion. For venous cannulation, the SVC is dissected free circumferentially, with the pericardial reflection being divided; the cannula is placed high on the vessel. The IVC is dissected free from the diaphragmatic reflection and cannulated low, as the IVC emerges from the diaphragm. The recipient heart is then explanted by transecting the SVC at the cavoatrial junction, and the IVC is divided with a generous cuff of right atrium. The left atrium is removed completely, leaving only two left atrial cuffs that include the ostia of the pulmonary veins on either side. The implantation of the donor's heart begins with the anastomosis of the left atrial cuff containing the left pulmonary veins to the corresponding orifice in the donor's left atrium. The right pulmonary veins are anastomosed in the same fashion. When both lungs are harvested, there may not be sufficient left atrial cuff for the cardiac allograft, in which case a modified technique can be used as described elsewhere. ${ }^{7}$ The SVC and the IVC are then anastomosed in an end-to-end fashion. Frequently, however, the donor heart IVC has been transected close to or at the right atrium, to provide sufficient length for a liver allograft. In these circumstances, a generous cuff of right atrium around the recipient IVC will facilitate the end-to-end anastomosis. The aorta and pulmonary artery are reconstructed in the usual manner.

Immunosuppressive prophylaxis. All patients received an induction course of murine monoclonal antibody muromonab-CD3 (5 mg intravenously daily) (OrthocloneOKT3; Ortho Biotech, Raritan, N.J.). The duration of this induction therapy changed from 14 to 10 days in November 1992 and has been 7 days since September 1993. All patients received an immunosuppressive regimen consisting of azathioprine $4 \mathrm{mg} / \mathrm{kg}$ intravenously before the operation and $2 \mathrm{mg} / \mathrm{kg}$ daily after the operation; methylprednisolone $500 \mathrm{mg}$ intravenously at the time of crossclamp removal and $125 \mathrm{mg}$ every 8 hours for three doses followed by oral prednisone $0.25 \mathrm{mg} / \mathrm{kg}$ daily during induction therapy, and thereafter $0.50 \mathrm{mg} / \mathrm{kg}$. Oral cyclosporine $5 \mathrm{mg} / \mathrm{kg}$ twice daily was started within 48 to 72 hours after the operation, after renal function had stabilized. Prednisone was tapered over a period of 6 months to 1 year, according to the results of endomyocardial biopsies.

Postoperative follow-up. Endomyocardial biopsy, right heart catheterization, and thermodilution cardiac output were obtained at specified intervals after the operation. ${ }^{8}$ Mitral and tricuspid regurgitation were determined by means of Doppler echocardiography. Hemodynamic and echocardiographic data were excluded if patients had acute cellular rejection (grade $\geq 1 \mathrm{~B}$ according to the criteria of the International Society for Heart and Lung Transplantation ${ }^{9}$ ) or humoral rejection. ${ }^{10,11}$

Statistical analysis. Freedom from an event (death or rejection) was analyzed by means of life table methods and actuarial curves were compared by means of the log-rank test. A Cox stepwise regression was used in multivariate analyses. For each patient, cardiac output and cardiac index values closest to 2 weeks, 6 months, and 1 year after the operation were selected and were compared by means of an analysis of variance.

Data are summarized as mean \pm standard deviation for continuous variables and as frequencies and percents for categorical values. All testing was two-sided using an $\alpha$ level of 0.05 , without adjustment for multiple testing.

\section{Results}

Preoperative recipient and donor characteristics. The mean age of the patients was $57.0 \pm 11.1$ years and the range from 14 to 77 years. Fifty-one patients were more than 60 years old (mean age of 65.4 years for this group). The majority of the patients were male $(92 \%)$ and white (82\%), $11 \%$ were black, $3 \%$ Hispanic, and $4 \%$ Asian. The diagnoses were almost equally distributed between ischemic (52\%) and idiopathic cardiomyopathy (44\%). Four patients had other diagnoses: sarcoidosis $(n=1)$, doxorubicin (Adriamycin) toxicity $(n=1)$, congenital heart disease $(n=1)$, and amyloidosis $(n=1)$. The mean left ventricular ejection fraction was $19.5 \%$ and the average pulmonary artery systolic pressure was $51.7 \pm 19.2 \mathrm{~mm} \mathrm{Hg}$. No patient was declined because of pulmonary hypertension, but patients with elevated pulmonary vascular resistance were treated with vasodilators, including intravenous nitroglycerin, nitroprusside, or prostaglandin $\mathrm{E}_{1}$ during the operative procedure and the perioperative period.

Donors were matched on the basis of blood type and body weight. The mean donor/recipient weight ratio was 0.92 . A weight mismatch of more than $20 \%$ (donor/recipient weight ratio $<0.8$ ) occurred in 28 patients $(28 \%)$.

Early (30-day) survival was $100 \%$ (Fig. 1). One- and 2-year survivals were $98 \% \pm 2 \%$ and $96 \% \pm 2 \%$, respectively. Recipient age and donor/recipient weight mismatch did not affect survival $(p>0.2$ for each of these parameters). The same applies to the duration of OKT3 therapy. The survival curves of patients with the three durations of OKT3 therapy are shown in Fig. 2. The seven late deaths were due to infections $(n=2)$, graft atherosclerosis $(n=3)$, acute rejection $(n=1)$, and nonspecific graft failure $(n=1)$. 


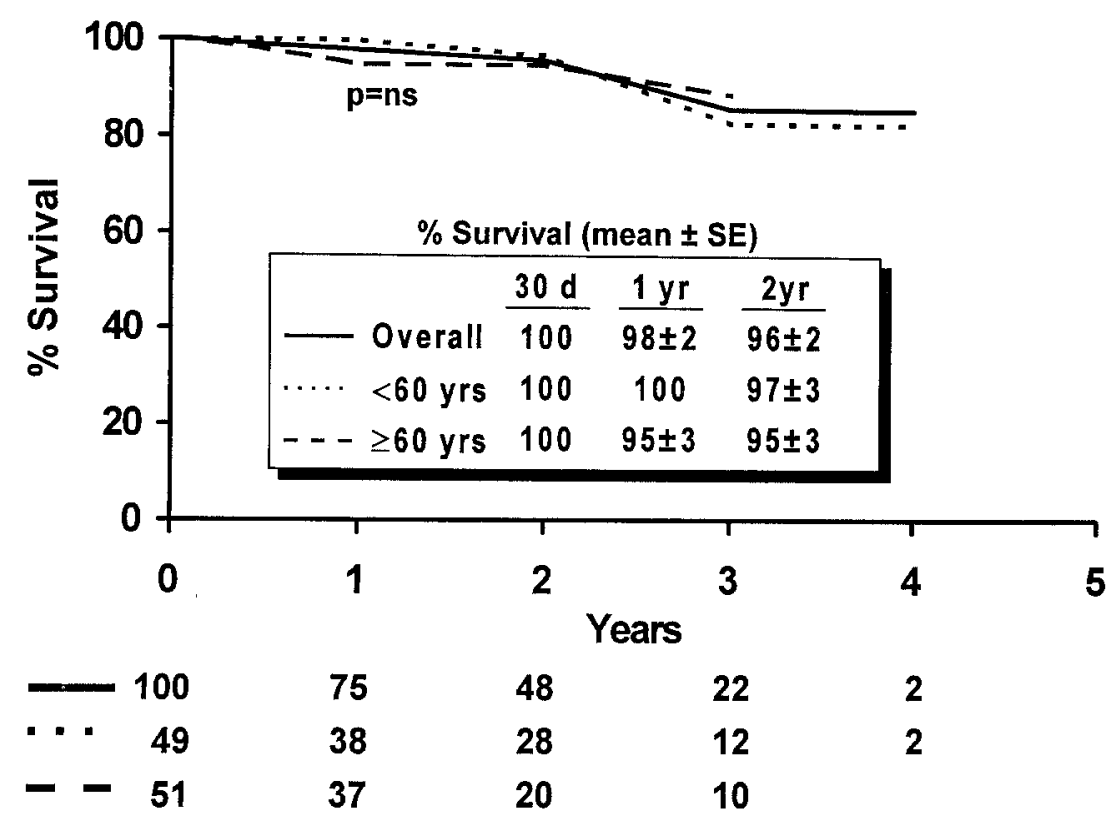

Fig. 1. Actuarial survival after orthotopic heart transplantation using bicaval and pulmonary venous anastomoses. Survival is shown for all patients and is also stratified according to the age of the recipient. Older ( $\geq 60$ years) and younger ( $<60$ years) patients did not differ significantly in survival. All mortalities were included in the analysis. The box inset indicates the probabilities of survival at 30 days, 1 year, and 2 years. Numbers below the figure indicate patients at risk during follow-up. $n s$, Not significant; $S E$, standard error.

Postoperative cardiac output. Cardiac output and cardiac index were analyzed at 2 weeks, 6 months, and 1 year after transplantation. When patients with differing durations of OKT3 therapy were stratified and compared (data not shown), patients with a longer duration of OKT3 therapy demonstrated increased values for cardiac output and cardiac index at 2 weeks $(p<0.001)$, but not at later evaluations.

Postoperative Doppler echocardiography. One year after transplantation, 50 patients had Doppler echocardiographic evaluation. Of these, 11 (22\%) had a degree of mitral regurgitation of $2+$ or greater (10 had $2+$ and one patient had $3+$; scale 0 to $4+$ ). Similarly, $18(36 \%)$ had $2+$ or more tricuspid regurgitation (16 patients had $2+$ and two patients had $3+$; scale 0 to $4+)$.

Treated rejection. Actuarial analysis of freedom from treated rejection is shown in Fig. 3, stratified according to the duration of OKT3 therapy. It is clear that treated rejection is less common in patients with only 7 days of therapy than in patients with 14 days of therapy $(p<0.0001)$.

Similarly, the duration of OKT3 therapy was strongly related to the number of episodes of rejec- tion in the first year after transplantation. Patients with 7 days of OKT3 therapy had a mean of 0.4 rejections in the first year, compared with 1.1 for patients with 14 days of OKT3 therapy $(p=0.0002)$.

Pacemaker placement. Insertion of a pacemaker was not required in any patient within the first 6 months after transplantation, and all the patients were discharged in normal sinus rhythm. One patient required permanent pacemaker implantation 7 months after the operation for high-grade atrioventricular block.

\section{Discussion}

The concept of "total" orthotopic heart transplantation with bicaval and pulmonary venous anastomoses was first introduced by Dreyfus, ${ }^{4}$ Yacoub, ${ }^{12}$ and their associates. The surgical technique used in our center differs from that used by other groups in two important aspects: first, a portion of azygos vein is incorporated into the SVC anastomosis, which may reduce the occurrence of anastomotic stenosis (none since this modification has been used); second, two small buttons of atrial tissue are excised with the left and right pulmonary veins, instead of one large atrial button, thus allowing more donor 


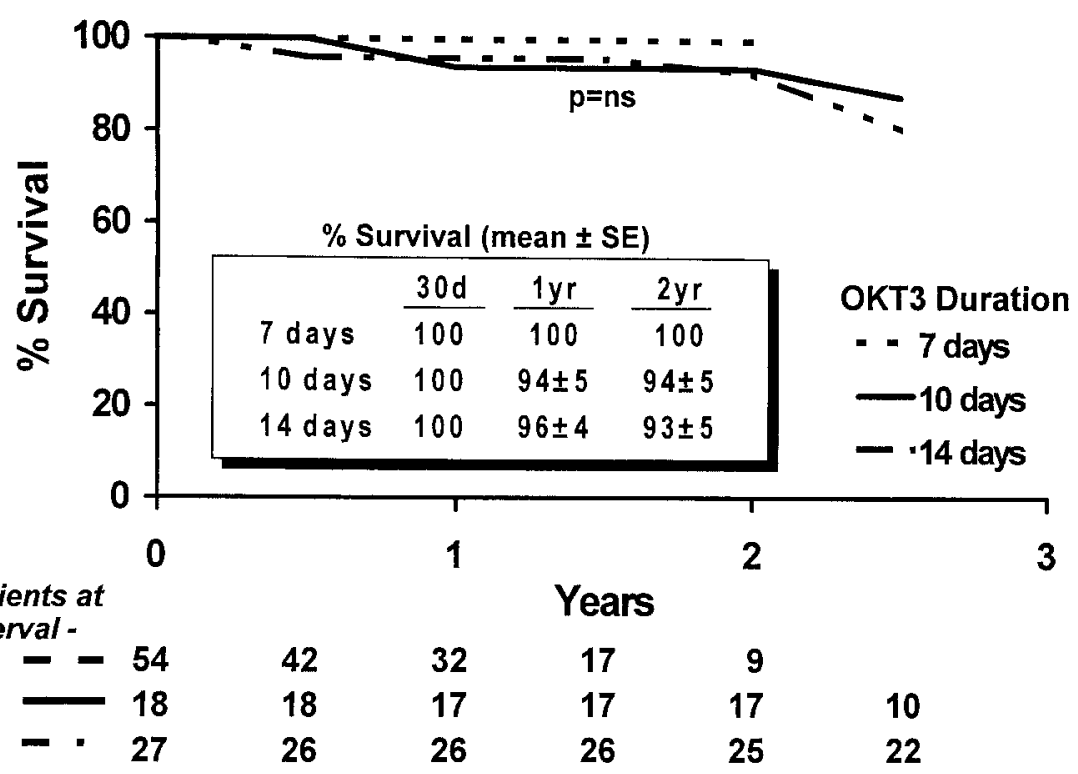

Fig. 2. Actuarial survival after orthotopic heart transplantation, stratified by duration of OKT3 induction therapy. There was no significant difference in survival among patients treated with the three durations of OKT3 therapy. All mortalities were included in the analysis. $n s$, Not significant; $S E$, standard error.

left atrial tissue to be transplanted, possibly resulting in better left atrial function.

We became interested in this technique because we noted a high incidence of permanent pacemaker requirement in the early postoperative period ${ }^{13}$ (14\% or nine of 64 patients) with the standard technique. Our initial intent to perform a randomized prospective trial comparing the traditional surgical technique with the new one was abandoned because of the uniform lack of need for permanent pacemaker insertion noted in the first 10 patients in whom the bicaval technique was used. For this reason, the decision was made to continue using the new technique so long as the patient was in normal sinus rhythm. This continues to be true for 124 consecutive patients, none of whom required a permanent pacemaker in the first 6 months after the operation and all of whom were discharged in normal sinus rhythm. This clear electrophysiologic advantage has been confirmed by other randomized and prospective trials. El Gamel and coworkers ${ }^{14}$ randomized 40 patients to the bicaval technique and 35 patients to the standard technique. A permanent pacemaker was required in three patients with the standard technique versus none with the bicaval technique. They also noticed that the patients in whom the bicaval technique was used had significantly fewer tachyarrhythmias (2/40 versus $11 / 35$ with standard technique). A similar incidence of atrial arrhythmias was found by Deleuze and colleagues ${ }^{15}$ in a randomized study. Retrospective studies by Brandt, Harringer, and $\mathrm{Hirt}^{16}$ and by Jeevanandam and coworkers ${ }^{17}$ have demonstrated a decreased incidence of atrial flutter and fibrillation and sinus node dysfunction and improved survival in patients in whom the bicaval technique was used.

From an electrophysiologic standpoint, we can conclude that sinus node dysfunction and atrial bradyarrhythmias necessitating pacemaker insertion, which were recognized early in the transplant experience ${ }^{18,19}$ and attributed to the atrial incision, surgical trauma to the sinus node, and surgical distortion of the atrium after implantation, have been almost completely eliminated by the bicaval technique.

Atrioventricular valve function in the posttransplantation period has been studied by Doppler echocardiography in several reports. A surprisingly high incidence of mitral regurgitation (13/20 patients, $60 \%)$ and tricuspid insufficiency (17/20 patients, $85 \%$ ) was observed by Angermann and associates $^{2}$ in patients in whom the standard technique was used. Stevenson and coworkers ${ }^{3}$ confirmed these findings. The current study reveals that at 1 year, the incidence of tricuspid and mitral regurgitation was substantially lower in our patients when 

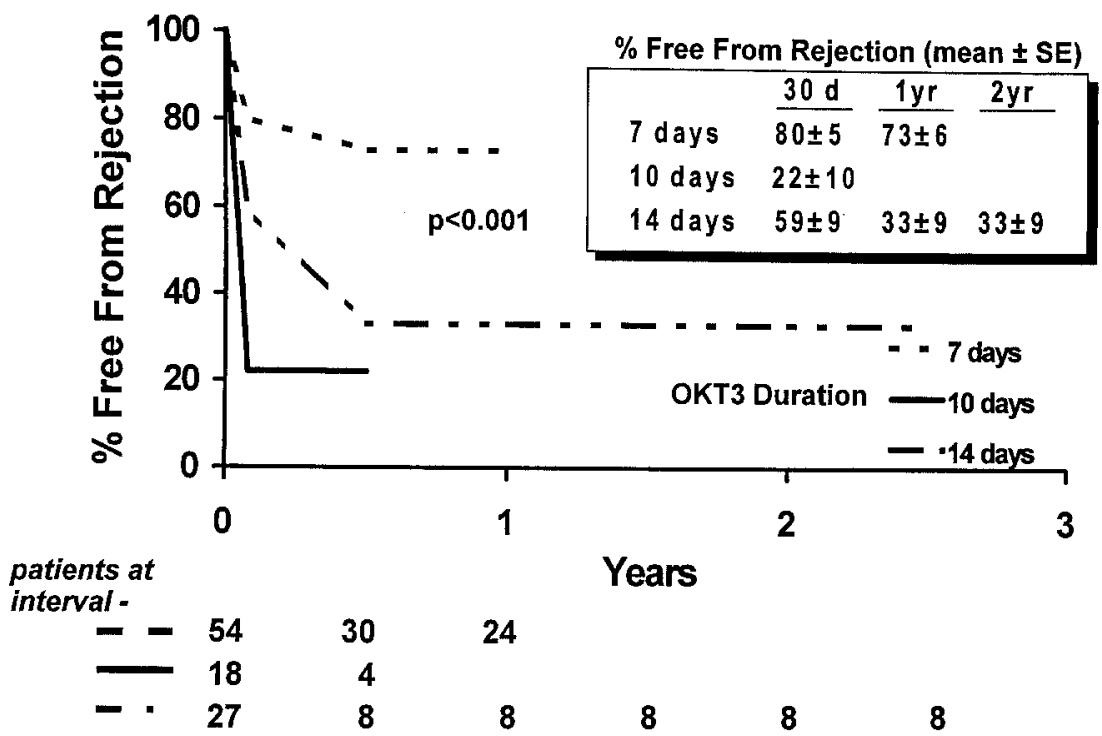

Years

24

8

$\begin{array}{lll}8 & 8 & 8\end{array}$

Fig. 3. Actuarial freedom from treated rejection according to duration of OKT3 therapy. There was significantly greater freedom from treated rejection in patients treated with 7 days of OKT3, in comparison with 14- and 10-day courses. For the entire population, freedom from treated rejection was $64 \% \pm 5 \%$ at 30 days, $51 \% \pm 5 \%$ at 1 year, and $49 \% \pm 5 \%$ at 2 and 3 years. $S E$, Standard error.

compared with the literature reports. This improvement in atrioventricular valve function in patients in whom the bicaval technique was used has been documented by other authors, ${ }^{5}$ who found less tricuspid regurgitation or less mitral regurgitation (or both) when these patients were compared with those in whom the standard technique was used. In one series by Leyh and associates, ${ }^{20}$ this difference became apparent only during exercise.

Atrial function after transplantation has also been the object of clinical and experimental investigation. In our institution, ${ }^{21}$ left atrial transport and function were evaluated by means of Doppler echocardiography in transplant recipients and normal control subjects. We found that left atrial transport and ejection force were similar in normal control subjects and patients undergoing the new transplantation technique, but were significantly lower in the standard technique group. In another study using magnetic resonance imaging, ${ }^{22}$ we found that the right and left atrial emptying fractions, as defined by the difference between atrial diastolic and systolic volumes divided by atrial diastolic volume, were significantly higher in the new technique group than in the standard technique group. Thus the bicaval and pulmonary venous anastomotic technique produces more physiologic atrial function than the biatrial technique, as evidenced by greater atrial ejection force and more normal atrial transport and atrial emptying fractions.

In an animal model, Goldstein and associates ${ }^{23}$ have shown that synchronous atrial contractility constitutes an important compensatory response to right ventricular dysfunction. This finding was confirmed in patients with right ventricular infarction who manifested severely compromised cardiac function with right ventricular asynchrony or depressed A waves (an indication of right atrial dysfunction). Recent experimental work from Bittner, Chen, and Kendall ${ }^{24}$ compares the two surgical techniques, with respect to atrial function, atrioventricular conduction, biventricular filling, and compliance in a dog model, using functional analysis, micromanometry, sonomicrometry, and ultrasonography. They conclude that atrial systole and right and left ventricular diastolic filling are better preserved with total orthotopic heart transplantation than with the standard technique.

The role of an intact right atrium in preventing the development of right ventricular failure after orthotopic heart transplantation, particularly in patients with marginally elevated pulmonary vascular resistance, is unknown. However, preliminary data from our institution ${ }^{25}$ show lower mean right atrial and pulmonary arterial pressures and improved cardiac output after transplantation performed by 
the bicaval technique (in comparison with the traditional technique) in patients with high preoperative pulmonary vascular resistance, indicating a lower risk of postoperative right ventricular failure with the bicaval technique.

During the course of the study, there was a change in our immunosuppression protocol. The duration of OKT3 therapy, in fact, was shortened from 14 to 7 days. To evaluate the impact of OKT3 duration on survival, the patients in whom the bicaval technique was used were grouped by duration of OKT3 therapy, and survival in the groups was compared by the log-rank test. Survival was not affected by the duration of OKT3 therapy, but the incidence of rejection was. As has been previously reported by us and by others, the patients with a 7-day course of induction therapy have an overall lower incidence of rejection because of a significantly lower incidence of humoral rejection, the incidence of cellular rejection being the same. Because the length of OKT3 therapy was also related to posttransplantation cardiac output, cardiac index, and mitral and tricuspid regurgitation, analysis of these latter outcomes was restricted to patients with 14 days of OKT3 induction therapy.

From our experience and from these studies, we can conclude that the bicaval and pulmonary venous anastomotic technique of total orthotopic heart transplantation, by preserving a more normal anatomic and geometric atrial configuration, has a clear electrophysiologic advantage, which is the elimination of the need for a permanent pacemaker. There is also a very low incidence of atrioventricular valve regurgitation. Clinical and experimental studies indicate that atrial function is improved with the bicaval technique and approaches that of a normal heart. The improved function conferred by the bicaval technique may be especially important in patients with high preoperative pulmonary vascular resistance and may lower the risk of postoperative right ventricular failure.

We thank Aurelio Chaux, MD, Wen Cheng, MD, and Gregory Fontana, MD, from the Division of Cardiothoracic Surgery, and Robert Frantz, MD, Arnold Friedman, MD, Harley Geller, MD, Erroll Hackner, MD, and Keith Kimble, MD, from Cardiothoracic Anesthesia, whose patients participated in this study. Special thanks to Soraya Radgar for preparation of the manuscript.

\section{REFERENCES}

1. Shumway NE, Lower RR, Stofer RC. Transplantation of the heart. Adv Surg 1966;2:265-84.
2. Angermann $\mathrm{CE}$, Spes $\mathrm{CH}$, Tammen A, et al. Anatomic characteristics and valvular function of the transplanted heart: transthoracic versus transesophageal echocardiographic findings. J Heart Transplant 1990;9:331-8.

3. Stevenson LW, Dadourian BJ, Kobashigawa J, Child JS, Clark SH, Laks H. Mitral regurgitation after cardiac transplantation. Am J Cardiol 1987;60:119-22.

4. Dreyfus G, Jebara V, Mihaileanu S, Carpentier AF. Total orthotopic heart transplantation: an alternative to the standard technique. Ann Thorac Surg 1991;52:1181-4.

5. Kendall WH, Ciulli F, Mullins PA, Biocina B, Dunning JJ, Large SR. Total orthotopic heart transplantation: an alternative to the standard technique (letter). Ann Thorac Surg 1992;54:187-8.

6. Blanche C, Czer LSC, Trento A, et al. Orthotopic heart transplantation with bicaval and pulmonary venous anastomoses. Ann Thorac Surg 1994;58:1505-9.

7. Blanche C, Czer LSC, Valenza M, Trento A. Alternative technique for orthotopic heart transplantation. Ann Thorac Surg 1994;57:765-7.

8. Valenza M, Czer LSC, Trento A, et al. Combined antiviral and immunoglobulin therapy as prophylaxis against cytomegalovirus infection after heart transplantation. J Heart Lung Transplant 1995;14:659-65.

9. Billingham ME, Cary NRB, Hammond EH, et al. A working formulation for the standardization of nomenclature in the diagnosis of heart and lung rejection: Heart Rejection Study Group. J Heart Transplant 1990;9:587-93.

10. Freimark D, Aleksic I, Trento A, et al. Hearts from donors with chronic alcohol use: a possible risk factor for death after heart transplantation. J Heart Lung Transplant 1996;15: 150-9.

11. Lones MA, Czer LSC, Trento A, Harasty D, Miller JM, Fishbein MC. Clinical-pathologic features of humoral rejection in cardiac allografts: a study in 81 consecutive patients. J Heart Lung Transplant 1995;14:151-62.

12. Yacoub M, Mankad P, Ledingham S. Donor procurement and surgical techniques for cardiac transplantation. Semin Thorac Cardiovase Surg 1990;2:153-61.

13. Blanche C, Czer LSC, Trento A, Fishbein MC, Doan D, Jordan $\mathrm{S}$, et al. Bradyarrhythmias requiring pacemaker implantation after orthotopic heart transplantation: association with rejection. J Heart Lung Transplant 1992;11:446-52.

14. El Gamel A, Yonan A, Deiraniya AK, et al. Orthotopic cardiac transplantation: comparison of standard and bicaval Wythenshawe techniques. J Thorac Cardiovasc Surg 1995; 109:721-30.

15. Deleuze PH, Benvenuti C, Mazzucotelli JP, et al. Orthotopic cardiac transplantation with direct caval anastomosis: Is it the optimal procedure? J Thorac Cardiovasc Surg 1995;109: 731-7.

16. Brandt M, Harringer W, Hirt SW. Bicaval vs. right atrial anastomosis in heart transplantation (abstract). J Heart Lung Transplant 1996;15:S51.

17. Jeevanandam V, Furukawa S, Prendergast TW, et al. Bicaval versus standard orthotopic heart transplantation: postoperative and long-term comparison (abstract). J Heart Lung Transplant 1996;15:S51.

18. Stinson EB, Dong E Jr, Iben A. Cardiac transplantation in man. Am J Surg 1969;118:182-7.

19. Barnard CN. What we have learned about heart transplants. J Thorac Cardiovasc Surg 1968;56:457. 
20. Leyh R, Jahnke W, Kraatz EG, et al. Cardiovascular dynamics and dimensions after bicaval and standard cardiac transplantation. Ann Thorac Surg 1995;59:1495-500.

21. Freimark D, Czer LSC, Trento A, et al. Improved left atrial transport and function with orthotopic heart transplantation by bicaval and pulmonary venous anastomoses. Am Heart J 1995;130:121-6.

22. Freimark D, Silverman JM, Aleksic I, et al. Atrial emptying with orthotopic heart transplantation using bicaval and pulmonary venous anastomoses: a magnetic resonance imaging study. J Am Coll Cardiol 1995;25:932-6.

23. Goldstein JA, Harada A, Yagi Y, et al. Hemodynamic importance of systolic ventricular interaction, augmented right atrial contractility and atrioventricular synchrony in acute right ventricular dysfunction. J Am Coll Cardiol 1990; 16:181-9.

24. Bittner HB, Chen EP, Kendall SW. Atrial systole and ventricular diastolic filling is more preserved in complete orthotopic cardiac transplantation compared to the standard technique (abstract). Annual meeting Society of Thoracic Surgeons 1996;B8:222-223.

25. Aleksic I, Czer LSC, Freimark D, et al. Improved resting hemodynamics after total vs. standard orthotopic heart transplantation in patients with high preoperative pulmonary vascular resistance (abstract). J Am Coll Cardiol 1996; 27(Suppl A):342A.

\section{Discussion}

Dr. Bartley P. Griffith (Pittsburgh, Pa.). I would like to comment on the evolution of this surgical technique, then to comment on the results of Dr. Trento's series, and finally to ask Dr. Trento three questions.

The technique of orthotopic cardiac transplantation began in the laboratories at Stanford and sprang into clinical acceptance through that group's work. Lower and Shumway have often been quoted for the technique, and it has withstood the test of time, with many patients benefiting. I suspect that the technique began to give way to a bicaval operation as opposed to a right atrial cuff technique when the domino procedure was introduced into cardiac transplantation. The potential benefits of the domino technique with bicaval anastomosis for the recipients were translated by the apparent reduction in atrial arrhythmia, and perhaps reduction in mitral regurgitation, as potentially a preferred method.

A number of groups subsequently studied bicaval anastomosis and incorporated the technique that Dr. Trento has just described-individual pulmonary vein anastomosis to the left atrium. Most notably, Mr. Yacoub and Dr. Dreyfus from Europe reported a series demonstrating this. Their results showed an increase in early cardiac output but most notably a small decrease in tricuspid regurgitation and a significant decrease in tricuspid regurgitation.

In the last few years Dr. Philippe DeLuz has also published a randomized study that includes an exercise component, bestowing the virtue of bicaval anastomosis and a small left atrial remnant. On the basis of that background, it would appear that Dr. Trento's group was well advised to enter into this trial.

I am struck by the stunning skill that Dr. Trento's group has demonstrated, not only with the technical aspects of this particular procedure, but also with the $100 \%$ inhospital survival. They demonstrate significant skill in their ability to select patients and care for them. I am going to disregard their references to the standard procedure, because these are noncontemporary control patients and probably represent a slightly different time era.

I have three questions for you, Dr. Trento. First, can you describe the proper performance of the SVC anastomosis? For instance, do you evert it? Have you seen any constrictions, and what do you about them? Have you had trouble fitting laterally displaced pulmonary veins removed from those patients who have a large left atrium back into the small donor heart that is to be placed? Second, is the early hemodynamic benefit that you propose the result of the preservation of sinus rhythm? If so, why is this benefit evanescent? Third, what is your explanation for the apparent reduction in mitral regurgitation with the total orthotopic transplant versus other less radical left atrial procedures? You have demonstrated that only 11 of 50 patients $(22 \%)$ have mitral regurgitation, and this is the lowest number that I have seen published. Do you think this is solely because of the independent pulmonary venous anastomosis and more complete preservation of left atrial anatomy?

Dr. Trento. Thank you for your comments, Dr. Griffith.

Early in our experience we did have one SVC stenosis. To avoid this complication, we transect the recipient SVC with $1 \mathrm{~mm}$ or $2 \mathrm{~mm}$ of right atrial cuff. We have used this technique in about 90 patients without any problem. We also used an azygos vein to enlarge the donor SVC stump. Regarding the surgical technique for the SVC anastomosis, we have tried the everting suture technique a few times, but we went back to the usual inverting, sewing from the inside technique, by using a very fine suture of 5 or 6-0 polypropylene and taking very small bites of tissue.

To answer your last question, regarding technical problems due to size discrepancy of the donor and the recipient's heart: If the IVC of the donor's heart is far away from the recipient's IVC stump, we align the right pulmonary veins, keeping in mind the IVC position. If we detect tension while we perform the IVC anastomosis, once the snaring tapes are released, the tension is completely released.

We do think that there is an early hemodynamic advantage with the new technique connected with the normal sinus rhythm in the patient. We have retrospectively examined our patients on the basis of their pulmonary vascular resistances, and we have seen that patients with high pulmonary vascular resistances have a lower right atrial pressure and pulmonary artery pressure with the bicaval technique than do the patients with the biatrial technique. Obviously these are retrospective reviews and their meaning is questionable.

Regarding the cause of mitral regurgitation, I think that by performing the individual pulmonary venous anastomosis you do obtain a normal-size left atrium. If you leave a posterior atrial cuff joining the two pulmonary veins, you will have a more enlarged left atrium with possibly abnormal atrial geometry. That is the only explanation that I can offer for the lower incidence of mitral regurgitation.

Dr. Gilles D. Dreyfus (Paris, France). I would like to congratulate Dr. Trento for these superb results, which we 
were unable to achieve. After Sir Magdi Yacoub, we had proposed in 1991 this modified orthotopic transplant technique after a presentation by Angermann showing many morphologic and physiologic imperfections of the standard technique at transesophageal echocardiography. Since then we have performed more than 50 of those transplantations, but we were unable to prove any hemodynamic superiority at the early phase when comparing the two techniques. What we have seen is that we could avoid thrombus formation, which can occur with the standard technique and may lead to strokes.

Unlike you, we have had one pacemaker implantation after the modified technique but have a significant decrease in the incidence of arrhythmias. I therefore have three questions. How do you explain that you did not require pacemaker implantation with the modified technique? Would you agree that many of the atrioventricular blocks that are encountered after orthotopic transplantation may be related to the preservation technique more than to the surgical technique? Do you believe that hemodynamic superiority of the complete technique as opposed to the standard technique, which could not be demonstrated at rest, probably could be demonstrated with exercise. Are you intending to try to prove that? Finally, you have clearly shown a reduced incidence of both mitral and tricuspid regurgitation. We have found the same result. However, this fate is denied by those who favor the bicaval technique as opposed to the standard technique. Could you explain the difference between those two techniques with regard to mitral regurgitation?

Dr. Trento. To answer your last question, the bicaval and pulmonary vein anastomosis technique leaves a normal left atrial anatomy. I think the absence of a pacemaker requirement in our experience is due to the fact that we do not touch any of the conduction tissue in the atrium. Our preservation technique is the same that was used 15 years ago-the Stanford solution-so I do not think it is a question of preservation. Yes, we are in the process of doing exercise testing to determine whether the hemodynamic advantage of the new technique is more evident during exercise. 